\title{
CREACIÓN AUTOMÁTICA DE SISTEMAS DE BÚSQUEDA DE RESPUESTAS EN DOMINIOS RESTRINGIDOS
}

Katia Vila, José-Norberto Mazón y Antonio Ferrández

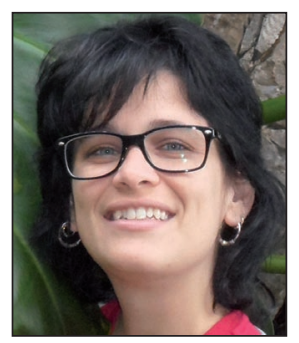

Katia Vila es doctora en ingeniería informática por la Universidad de Alicante. Ha sido profesora asistente en el Departamento de Informática de la Universidad de Matanzas en Cuba, impartiendo docencia en ingeniería informática en asignaturas relacionadas con la inteligencia artificial, la programación descriptiva, y la recuperación de información. Es miembro del Grupo de investigación en Procesamiento del Lenguaje y Sistemas de Información (GPLSI) del Departamento de Lenguaje y Sistemas Informáticos de la Universidad de Alicante. Ha publicado diferentes trabajos sobre sistemas de búsqueda de respuestas (BR). Disfruta de una beca MAEC-AECID (España) para un proyecto post-doctoral sobre sistemas de BR.

Universidad de Alicante Departamento de Lenguajes y Sistemas Informáticos Apartado de Correos 99. 03080 Alicante kvila@dlsi.ua.es

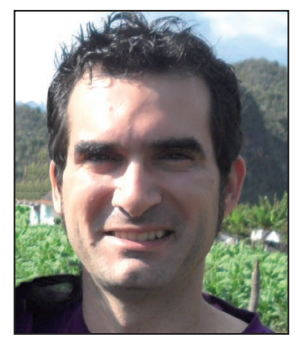

José-Norberto Mazón es profesor ayudante doctor en el Departamento de Lenguajes y Sistemas Informáticos de la Universidad de Alicante. Ha publicado varios trabajos en revistas y conferencias especializados de carácter internacional (como ER, ICCS, JISBD, DSS, DKE, etc.). Ha realizado estancias de investigación en la Universidad de Münster (Alemania) y la Universidad de Toronto (Canadá). Su investigación se centra principalmente en inteligencia de negocio y desarrollo de software dirigido por modelos. Ha participado en la organización de varias ediciones de los talleres "Business intelligence and the Web" (Beweb) y "Web and requirement engineering" (WeRE).

Universidad de Alicante Departamento de Lenguajes y Sistemas Informáticos Apartado de Correos, 99. 03080 Alicante jnmazon@dlsi.ua.es

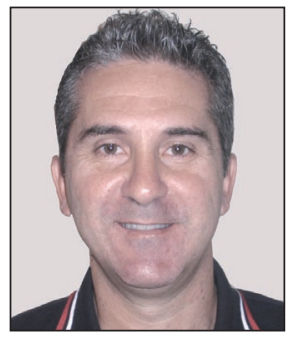

Antonio Ferrández es profesor en el Departamento de Lenguajes y Sistemas Informáticos de la Universidad de Alicante. Obtuvo su doctorado en informática en la misma universidad. Sus intereses de investigación son: procesamiento del lenguaje natural, resolución de problemas lingüísticos (anáfora o elipsis), extracción de información, sistemas de recuperación de información y búsqueda de respuestas. Ha participado en proyectos, acuerdos con empresas privadas y organismos públicos relacionados con sus temas de investigación. Ha dirigido tesis doctorales y es autor de artículos de revista y ponencias de congreso.

Universidad de Alicante Departamento de Lenguajes y Sistemas Informáticos Apartado de Correos, 99. 03080 Alicante http://www.dlsi.ua.es/ antonio/antonio.htm/ antonio@dlsi.ua.es

\section{Resumen}

Los sistemas de búsqueda de respuestas (BR) se pueden considerar como potenciales sucesores de los buscadores tradicionales de información en la Web. Para que sean precisos deben adaptarse a dominios concretos mediante el uso de recursos semánticos adecuados. La adaptación no es una tarea trivial, ya que deben integrarse e incorporarse a sistemas de BR existentes varios recursos heterogéneos relacionados con un dominio restringido. Se presenta la herramienta Maraqa, cuya novedad radica en el uso de técnicas de ingeniería del software, como el desarrollo dirigido por modelos, para automatizar dicho proceso de adaptación a dominios restringidos. Se ha evaluado Maraqa mediante una serie de experimentos (sobre el dominio agrícola) que demuestran su viabilidad, mejorando en un 29,5\% la precisión del sistema adaptado.

\section{Palabras clave}

Búsqueda de respuestas, Recuperación de información, Dominios restringidos, Desarrollo dirigido por modelos.

\section{Title: Automatic generation of question answering systems in restricted domains}




\begin{abstract}
Question answering (QA) applications can be considered as the potential successors to the traditional information retrieval on the Web. However, QA systems should be adapted to restricted domains for the sake of precision. This adaptation is not a trivial task, since several heterogeneous resources related to a restricted domain must be integrated into existing QA systems. This paper presents the Maraqa tool, whose novelty is the use of software engineering techniques such as model driven development to automate the adaptation process. It is worth noting that Maraqa has been evaluated through a set of experiments (within the agricultural domain) that demonstrate its applicability: the precision of the adapted QA system showed $29.5 \%$ improvement.
\end{abstract}

\title{
Keywords
}

Question answering, Information retrieval, Restricted domains, Model driven development.

Vila, Katia; Mazón, José-Norberto; Ferrández, Antonio. “Creación automática de sistemas de búsqueda de respuestas en dominios restringidos". El profesional de la información, 2012, enero-febrero, v. 21, n. 1, pp. 16-26.

http://dx.doi.org/10.3145/epi.2012.ene.03

\section{Introducción y motivación}

Con la sobreabundancia de información y la heterogeneidad en los formatos de acceso es complicado localizar informaciones concretas. Los usuarios necesitan sistemas que les permitan acceder a información precisa desde los diferentes recursos existentes de una forma transparente y simple.

La primera solución aportada por la comunidad científica para conseguir un acceso sencillo y rápido a la inconmensurable cantidad de información digital accesible, fue la recuperación de información (RI) o information retrieval (BaezaYates; Ribeiro-Neto, 1999). La RI consiste en la selección en un depósito de documentos de los que tengan mayor relevancia para una consulta realizada por un usuario.

\section{La BR tiene como objetivo obtener res- puestas a preguntas precisas formuladas en lenguaje natural}

Los sistemas de RI más conocidos son los que actúan sobre internet y localizan información en la Web, por ejemplo los motores de búsqueda como Google o Yahoo. Según Russell y Norvig (2003), este modelo de RI corresponde casi totalmente a palabras, ya que acepta una cantidad mínima de sintaxis (palabras que deben aparecer una junto a la otra), y un papel diminuto de clases semánticas (en forma de listas de sinónimos). Por ello los resultados son en muchas ocasiones documentos que contienen los términos de la consulta, pero no son la información deseada. Por otro lado, la salida es una lista de documentos ordenada en función de medidas de similitud con la pregunta y medidas indirectas de la visibilidad y prestigio de la información (por ejemplo page rank de la web), lo cual puede convertir a esos motores en sistemas manipulables. Luego resta una ardua tarea ya que el usuario debe revisar y leer cada documento de la lista obtenida, lo primero para ver si en realidad está relacionado con los requerimientos solicitados y lo segundo para localizar en su interior la información puntual que se desea.

http://www.google.com

http://www.yahoo.com
Los inconvenientes mencionados impulsaron la investigación en sistemas de búsqueda de respuesta (BR) o question answering, que tienen como objetivo la obtención de respuestas concretas a preguntas precisas indicadas por el usuario directamente en lenguaje natural.

En las figuras 1 y 2 se muestran las salidas para la pregunta What year did Wilt Chamberlain score 100 points? de un sistema de RI como Google y de un sistema de BR como NSIR respectivamente. En el caso de la RI se aprecia, en contraste con la BR, que el usuario ha de buscar dentro de los documentos devueltos.

http://clair.si.umich.edu/clair/NSIR/html/nsir.cgi

Los sistemas de BR deben adquirir un nivel de comprensión del texto muy superior al alcanzado por la RI, por lo que es habitual realizar un análisis léxico, sintáctico y semántico tanto de la pregunta como de los documentos. Este tipo de análisis conlleva un coste computacional muy elevado, el cual se supera mediante el filtrado de los documentos a través de la RI, es decir, entre los millones de documentos de partida, la BR realiza el análisis y búsqueda de la respuesta únicamente sobre unos cientos de pasajes (conjunto de oraciones de un documento).

Por último, se analizan las diferencias entre BR y RI desde el punto de vista de la propia pregunta que realiza el usuario. En primer lugar, habitualmente la pregunta en RI no es más que una serie de palabras ("invención teléfono"), mientras que en BR ésta ha de ser una pregunta formulada correctamente en lenguaje natural (“¿Cuándo fue inventado el teléfono?"). En segundo lugar, la RI se centra en la propia pregunta, mientras que la BR se centra en la respuesta a dicha pregunta. Por ejemplo, si planteamos la pregunta “¿Cuándo fue inventado el teléfono?" a un sistema de RI, buscará el documento que más veces contenga "teléfono", mientras que la BR buscará el que contenga la fecha concreta de la invención del teléfono.

La investigación en BR se ha visto incentivada a partir de 1999 por conferencias como TREC (Text retrieval conference) desde su octava edición, CLEF (Cross-language evaluation forum) y Ntcir (NII Test collection for IR systems). En estas conferencias compiten sistemas de BR desarrollados tanto por instituciones académicas como por empresas. Su 


\begin{tabular}{|c|c|c|}
\hline Google & What year did Wilt Chamberlain score 100 points? & Q \\
\hline & & Búsqueda avanzada \\
\hline Búsqueda & Aproximadamente 474.000 resultados $(0,38$ segundos $)$ & \\
\hline Todo & Wilt Chamberlain's 100-point game - Wikipedia, the free encyclopedia & \\
\hline Imágenes & $\begin{array}{l}\text { en wikipedia.org/...WVilt_Chamberlain's_100-poi.... Traducir esta página } \\
\text { Wilt Chamberlain's 100-point game, named by the National Basketball ... however, for }\end{array}$ & \\
\hline Maps & $\begin{array}{l}\text { the } 100 \text { points scored by Warriors center Wilt Chamberlain. ... triple- and quadruple- } \\
\text { teamed by the Knicks, who did not shy away from hard fouls to distract the center. .... }\end{array}$ & \\
\hline Videos & Over the years, Harvey Pollack, who at the time was in charge of both ... & \\
\hline Noticias & Wilt Chamberlain - Wikipedia, the free encyclopedia & \\
\hline Shopping & en.wikipedia.org/wiki/Wilt_Chamberlain - Traducir esta página & \\
\hline Foros de debate & — Mostrar más resultados de wikipedia. org & \\
\hline Más & $\begin{array}{l}\text { How did Wilt Chamberlain score } 100 \text { Points in one game? - Yahoo ... } \\
\text { answers.yahoo.com/... findex?qid... - Estados Unidos - Traducir esta página }\end{array}$ & \\
\hline Alicante & 19 respuestas - 28 Nov 2007 & \\
\hline Cambiar ubicación & $\begin{array}{l}\text { How did Wilt Chamberlain score } 100 \text { Points in one game? ... The game has changed } \\
\text { over the years and now we have loads of talent on per ... }\end{array}$ & \\
\hline Wha & $\begin{array}{l}\text { What it called when Wilt Chamberlain scored } 100 \text { points in one game ... } \\
\text { What team did he play? when wilt chamberlain score } 100 \text { points } \ldots\end{array}$ & \\
\hline Páginas en español & Is Wilt Chamberlain's 100-point game the most hardest record to ... & \\
\hline Páginas de España & Más resultados de answers.yahoo.com » & \\
\hline
\end{tabular}

Figura 1. Salida de un sistema de RI para una pregunta en lenguaje natural

\begin{tabular}{|l|l|l|}
\hline \multicolumn{1}{|c|}{ Sample Questions } & \multicolumn{1}{|c|}{ Type your question below } \\
or choose from IREC-8 TREC.9
\end{tabular}

Figura 2. Salida de un sistema de BR para una pregunta en lenguaje natural

funcionamiento general (dependiendo del tipo de subtarea sobre la que se compite) consiste en que los participantes disponen de unas colecciones de documentos y una serie de preguntas, cuya respuesta es analizada por un comité que ordena cada sistema por una serie de medidas. Estas colecciones de documentos suelen ser de un tamaño considerable y de contenidos diversos conocidos como de "dominio abierto" (DA).

http://trec.nist.gov

http://www.clef-initiative.eu

http://research.nii.ac.jp/ntcir/index-en.html

Algunos ejemplos de sistemas de BR con una dilatada trayectoria son Start (SynTactic analysis using reversible transformations) y Wolfram Alpha.

Start, operativo desde diciembre de 1993, fue realizado por Boris Katz y los componentes del InfoLab Group del MIT Computer Science and Artificial Intelligence Laboratory (Katz, 1997). Está basado en el etiquetado previo de segmentos de texto según diferentes granularidades (Katz et al., 2006), lo cual facilita el acceso a los tipos de respuesta predefinidos, pero presenta la desventaja de limitar los tipos de pregunta a realizar al sistema.

http://start.csail.mit.edu

Siguiendo la misma estrategia de Start, Wolfram Alpha (anunciado en marzo de 2009) -de la compañía Wolfram Research, presidida por Stephen Wolfram-, extrae respues- tas de una base de datos estructurada. Este sistema indexa una gran cantidad de respuestas, sobre las cuales realiza un proceso de inferencia cuando se plantea una pregunta. Es decir, ha construido una gran base de datos de conocimiento que utiliza para este proceso de inferencia. De un modo similar funciona $C y c$, el cual aúna una ontología y una base de datos de conocimiento general para llevar a cabo razonamientos de tipo humano. Este tipo de planteamientos adoptados tradicionalmente tienen limitaciones de extensibilidad debido a la estructuración predefinida de los tipos de respuestas.

http://www.wolframalpha.com http://www.cyc.com

A pesar de los amplios esfuerzos investigadores realizados, las carencias de los sistemas de RI actuales y el acuerdo general de que los sistemas de BR son sus potenciales sucesores, no se ha generalizado su uso. El motivo principal es que la BR no es lo suficientemente precisa sobre un dominio abierto para el coste computacional que supone, siendo necesarios muchos recursos semánticos y una capacidad de razonamiento no alcanzada de momento.

Estos objetivos sí se podrían alcanzar sobre "dominios restringidos" (DR) tales como el dominio médico o legal, en los que se disponga de recursos semánticos suficientes.

\section{A pesar de las investigaciones, las caren- cias de los sistemas de RI y la creencia de que los sistemas de BR son sus suceso- res, no se ha generalizado su uso}

Nuestro trabajo se centra en la adaptación automática de sistemas de BR en DA a dominios restringidos. Se presenta la herramienta Maraqa (Model-driven adaptation for restricted-domain question answering), que automatiza el proceso de adaptación y por tanto, puede usarse como un recurso que otorgará a todo sistema de BR en DA la capacidad para enfrentar el cambio, para introducir y extraer información de diferentes dominios restringidos independientemente del idioma y formato de sus recursos de conocimiento. Para ello, nos basaremos en el desarrollo dirigido por modelos o model-driven development (Bézivin, 2005).

\section{Arquitectura de un sistema de BR}

Después de estudiar varias de las aproximaciones de BR presentadas en conferencias como TREC y CLEF, se ha observado que la mayoría de ellas tiene una arquitectura común que se muestra en la figura 3. 
El módulo de indexación incluye los procesos aplicados a la colección de documentos de manera offline con el objetivo de acelerar el proceso de BR:

- indexación de documentos para el sistema de $\mathrm{RI}$, que usa información estadística (se asignan pesos a las palabras);

- indexación de documentos para el proceso de BR, que usa información léxica, sintáctica y semántica.

Por otro lado el módulo de búsqueda es el encargado de realizar 3 procesos para responder a la pregunta del usuario:

1. Análisis de la pregunta, en el cual

- se clasifica la pregunta [ej.: en función del tipo de respuesta esperada (TRE), que nos indica el

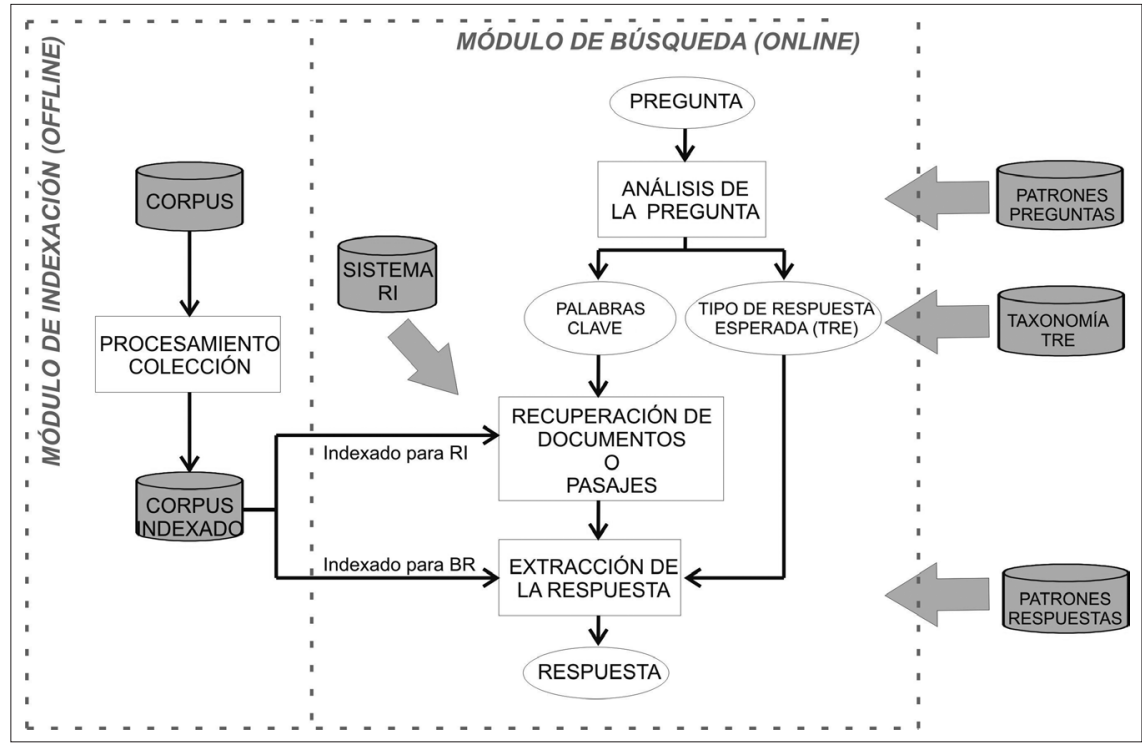

Figura 3. Arquitectura típica de un sistema de BR tipo semántico de la información a buscar, como "tipo persona” para la pregunta “¿Quién es el presidente de España?"];

- se extraen las palabras clave de la pregunta para usarlas como consulta en el siguiente proceso de RI (ej.: para "¿Cuál es el correo del Ministerio de Economía y Competitividad?", es habitual descartar el término "correo" porque éste no suele aparecer en la respuesta y su inclusión en la pregunta de RI podría devolver pasajes que contengan dicho término en lugar de la información buscada. Esto es lo que ocurre en la figura 1, en la que se muestran pasajes con las apariciones del término year para la pregunta What year did Wilt Chamberlain score 100 points?).

2. Recuperación de documentos o pasajes relevantes, donde se utilizan las palabras clave previamente detectadas y el indexado de los documentos para la RI realizado con anterioridad, como entradas del sistema de RI. La salida serán los documentos o pasajes relevantes para la pregunta del usuario, reduciendo así el espacio de búsqueda para la BR.

3. Análisis del conjunto de documentos o pasajes relevantes usando el indexado para la BR previamente realizado, con la finalidad de encontrar la respuesta esperada por el usuario.

\section{Problemas en la adaptación de un sistema de BR de DA a DR}

Si analizamos la arquitectura de un sistema de BR es obvio que las fases de análisis de la pregunta y extracción de la respuesta son dependientes del conocimiento, usualmente incluido en patrones, que se tenga del dominio de aplicación. En el contexto de este trabajo, se entiende por "patrones" (Vila, 2010) todas las posibles estrategias para la detección de las relaciones entre los elementos de la pregunta y la respuesta (ej.: formas lógicas, expresiones regulares, relaciones sintácticas, relaciones de dependencia, etc.). Además se puede destacar la repercusión que tiene la fase de análisis de la pregunta en el resto de fases de la arquitectura típica de un sistema de BR y por tanto, en la eficiencia del sistema.

En esta fase es crucial la determinación del tipo semántico de la respuesta que espera el usuario o TRE, por medio de una taxonomía de TRE [también conocida como jerarquía de preguntas (Li; Roth, 2006) u ontología de preguntas (Metzler; Croft, 2005)] predefinida. Una correcta especificación de la taxonomía de TRE implica una efectiva detección del TRE de la pregunta del usuario, reduciendo así el espacio de búsqueda de las respuestas candidatas y brindando una respuesta más precisa (Li; Roth, 2006; Hovy; Hermjakob; Ravichandran, 2002). Un ejemplo ilustrativo sería para la pregunta del usuario "¿Quién es el presidente de España?”, el TRE sería "persona" y las respuestas candidatas serían nombres propios como "Mariano Rajoy".

\section{Para el funcionamiento de un sistema} de $B R$ de $D A$ en dominios restringidos es necesaria la adaptación de sus patrones de preguntas y respuestas y de la taxonomía de TRE que se utilice

Teniendo en cuenta estas consideraciones, se puede afirmar que para lograr el correcto funcionamiento de un sistema de BR de DA en dominios restringidos es necesaria la adaptación de:

- los patrones de preguntas y respuestas del sistema de $\mathrm{BR}$;

- la taxonomía de TRE que se utilice.

Sin embargo, en las aproximaciones actuales para alcanzar dicha adaptación persisten dos problemas fundamentales:

- adaptación manual de los patrones de BR (Ferrés; Rodríguez, 2006; Peñas et al., 2009) y las taxonomías de TRE (Sekine; Sudo; Nobata, 2002; Hovy; Hermjakob; Ravichandran, 2002; Metzler; Croft, 2005; Li; Roth, 2006) a dominio restringidos, lo cual requiere de un esfuerzo enorme en función del tiempo y del coste propios de la complejidad inherente de los conceptos provenientes de esos dominios (Mollá; Vicedo, 2007); 
- definición de los patrones de BR y las taxonomías de TRE para dominios restringidos por medio del análisis de corpus de preguntas-respuestas potenciales (Terol; MartínezBarco; Palomar, 2006; Kosseim; Yousefi, 2008), lo que no es una situación realista ya que es muy difícil y compleja la adquisición de este tipo de corpus en dominios restringidos.

Después de analizar la situación actual de adaptación de sistemas de BR en DA a dominios restringidos, parece indiscutible la necesidad de diseñar estrategias que faciliten este proceso. Principalmente, las propuestas deben:

- elevar el grado de automatización de la adaptación, evitando que sea un proceso tedioso y complejo;

- explotar los recursos de conocimiento disponibles para un dominio concreto independientemente de su esquema de representación;

- utilizar el corpus textual como punto de partida y fuente de información principal, en detrimento del (poco realista en dominios restringidos) uso de corpus de preguntas;

- aumentar la precisión de la BR.

Para alcanzar estos objetivos, presentamos Maraqa, una herramienta que permite una adaptación sistemática de los sistemas de BR en DA a nuevos dominios restringidos basada en el paradigma de ingeniería del software conocido como desarrollo dirigido por modelos (model driven development, $M D D)$, que se basan en la representación de los conceptos y actividades que rigen un área concreta de conocimiento en lugar de usar terminología informática o codificación de algoritmos en lenguajes de programación complejos (Mellor et al., 2003). La creación de estos modelos debe basarse en una sintaxis gráfica, mediante reglas y normas establecidas en un metamodelo (Kleppe et al., 2003). Además, mediante el uso de $M D D$ se posibilita la generación automática de la aplicación software a partir de modelos (Mellor; Clark; Futagami, 2003). Por lo tanto, MDD enfatiza dos aspectos clave: los modelos y las transformaciones entre ellos para llegar a obtener el código fuente correspondiente del sistema software.

\section{Maraqa}

Ha sido desarrollado siguiendo el paradigma MDD mediante la plataforma Eclipse. Permite adaptar fácilmente sistemas de BR en DA a dominios restringidos, haciendo a estos sistemas útiles para su uso en dominios técnicos y no triviales que necesitan de una alta precisión como medicina, farmacia, derecho, etc. Por ejemplo, en el dominio agrícola una pregunta tipo podría ser "¿Qué enzima incrementa la digestibilidad del fósforo orgánico en los animales?". La pregunta es sobre un dominio restringido por lo que su terminología es más específica y por lo tanto es incorrectamente respondida por sistemas de BR en DA. En el trabajo de Vila et al. (2011) se realizó una serie de experimentos para comparar la precisión de un sistema de BR de DA desarrollado en la Universidad de Alicante, Ilamado AliQAn (Roger et al., 2008) y su adaptación al dominio agrícola mediante Maraqa. Los resultados indicaron que la precisión del sistema de BR AliQAn fue de $28,8 \%$ comparado con la media del sistema en un dominio abierto que es alrededor del $43 \%$ de precisión. Una vez AliQAn fue adaptado usando Maraqa, la precisión alcanzó un 58,3\% (Vila; Mazón; Ferrández, 2011). En el apartado de evaluación de Maraqa se describen con mayor profundidad los experimentos realizados.

http://www.eclipse.org

Maraqa permite adaptar sistemas de BR en dominios abiertos a dominios restringidos

Para adaptar sistemas de BR a DR, Maraqa requiere los siguientes recursos:

a)Conocimiento no estructurado: en este caso se refiere a toda la documentación no estructurada disponible en el dominio restringido al cual se desea adaptar el sistema de BR. Esta documentación es conocida como colección de documentos o corpus.

b)Conocimiento semi-estructurado o estructurado: cualquier sistema de organización del conocimiento (SOC) -llamados en inglés knowledge organization systems, KOS (Hodge, 2000)-, disponible en el dominio. Estos recursos de conocimiento incluyen una variedad de esquemas para organizar, manejar y recuperar información. El término SOC pretende abarcar cualquier tipo de esquema que sirva para gestionar el conocimiento, como diccionarios, taxonomías, tesauros, ontologías, etc. Según las áreas de conocimiento a las que se refiera un SOC, existen dos tipos: SOC genérico como WordNet, EuroWordNet, SUMO; o SOC de dominio, tales como el tesauro Agrovoc para el dominio agrícola, el tesauro multilingüe EuroVoc que abarca la terminología de los ámbitos de actividad de la Unión Europea, el metatesauro UMLS para el dominio médico, etc.

http://wordnet.princeton.edu

http://www.illc.uva.nl/EuroWordNet

http://www.ontologyportal.org

http://www.fao.org/agrovoc

http://eurovoc.europa.eu

http://www.nlm.nih.gov/research/um/s/knowledge_ sources/metathesaurus

c) Código fuente de los patrones de preguntas y respuestas del sistema de BR en DA que se desea adaptar.

Cabe resaltar que Maraqa es independiente del idioma de los recursos que emplee, pero para su correcto funcionamiento todos estos recursos deben estar en el mismo idioma.

A partir de estos recursos, Maraqa obtiene:

- taxonomía de TRE refinada para el dominio en cuestión;

- código fuente de los patrones de preguntas adaptados al dominio;

- código fuente de los patrones de respuesta adaptados al dominio.

Para explicar la herramienta utilizaremos como caso de estudio la pregunta "¿Qué glicósidos tienen un efecto defaunante en el rumen?". Nuestro objetivo sería adaptar AliQAn a este dominio agrícola. Para ello se necesita, en primer lugar, un corpus o conocimiento no estructurado, sobre el 


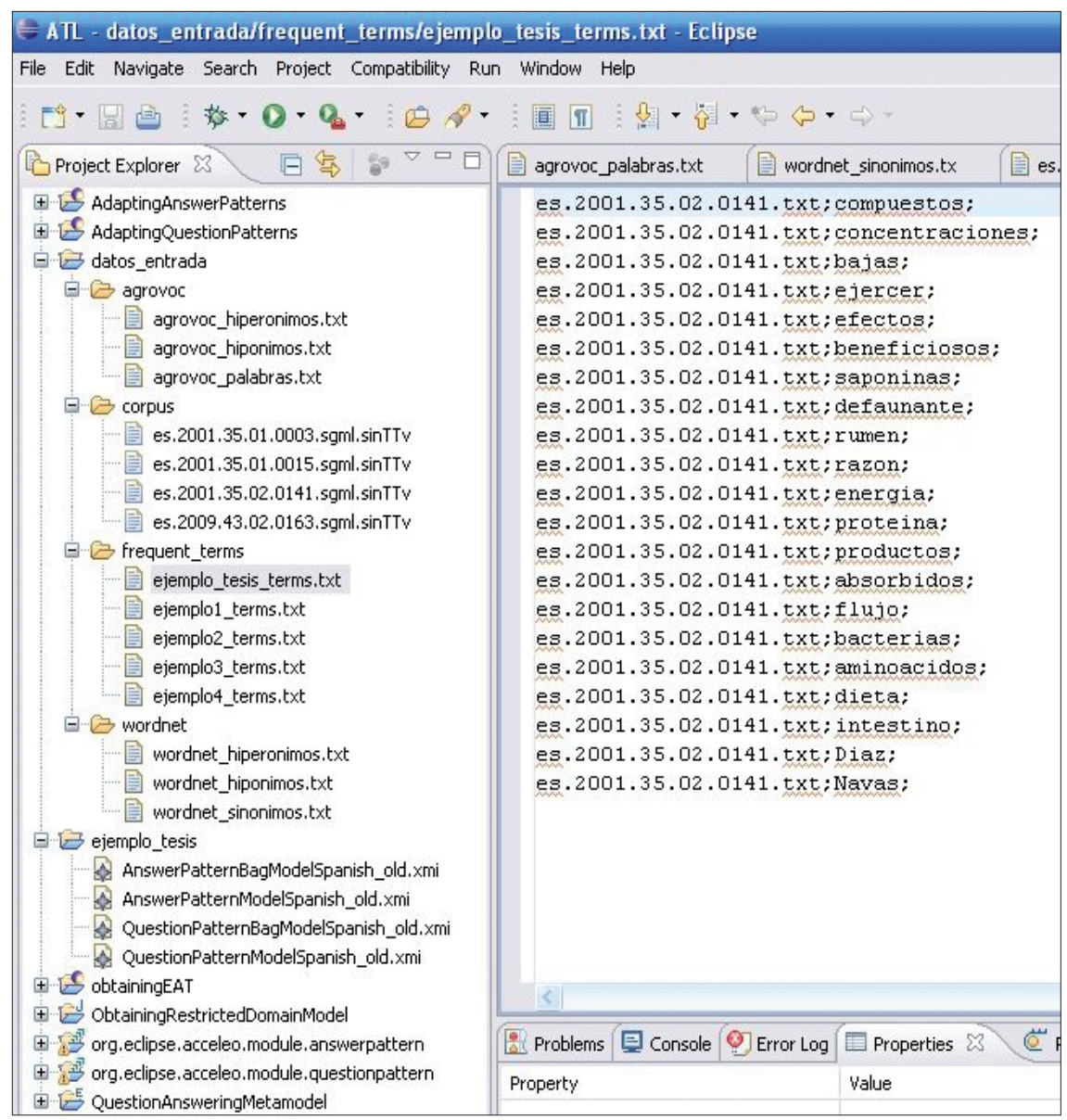

Figura 4. Obtención de términos más relevantes

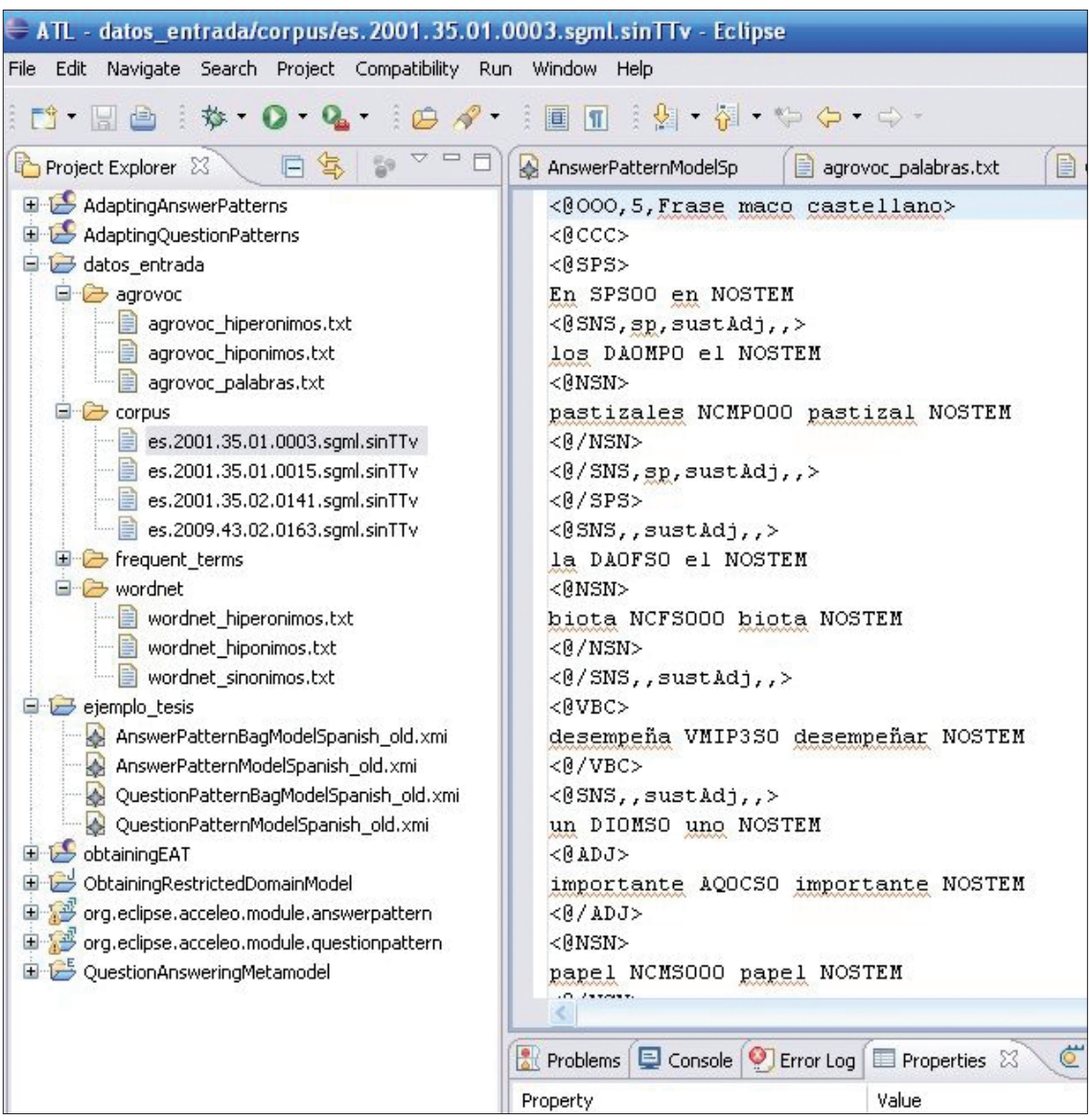

Figura 5. Ejemplo de relaciones sintácticas entre términos relevantes. dominio. En este caso nuestro corpus es un conjunto de artículos de investigación de la Revista cubana de ciencia agrícola (RCCA). En este corpus se encuentra el fragmento de texto que contiene una respuesta candidata: "[...] Sin embargo, en ocasiones, estos compuestos, en concentraciones bajas, pueden ejercer efectos beneficiosos. Por ejemplo, las saponinas tienen un efecto defaunante en el rumen, lo que puede contribuir al aumento de la razón energía: proteína de los productos absorbidos debido al incremento del flujo de bacterias y de aminoácidos de la dieta hacia el intestino [...]". http://www.ica.inf.cu/revista-cubanade-ciencia-agricola/index.php http://redalyc.uaemex.mx/src/

El primer paso realizado por Maraqa consiste en determinar los términos más relevantes del dominio a partir de la indexación del corpus (para RI y BR según la figura 3), tomando los términos que sean sustantivos, verbos $\mathrm{y}$ adjetivos y que tengan una determinada frecuencia de aparición en el corpus. Para nuestro caso, los términos más relevantes se pueden ver en la captura de pantalla de Maraqa de la figura 4 precedidos por el documento en el que aparecen (ej.: "compuestos" que aparece en el documento "es.2001.35.02.0141.txt").

Se obtienen las relaciones entre estos términos más relevantes usando el análisis sintáctico del corpus realizado con anterioridad en el indexado para BR (ver fase offline de figura 3); por medio de herramientas de procesamiento del lenguaje natural: primero se utilizó el etiquetador léxico o PoS tagger MACO (Acebo et al., 1994) y luego el analizador sintáctico Supar (Ferrández; Palomar; Moreno, 1999). Por ejemplo, si un sustantivo puede ser sujeto de un verbo o qué adjetivos modifican a un sustantivo. En la figura 5 se observa un ejemplo de este análisis con Maraqa (la relación adjetivo-sustantivo entre los términos "importante-papel", o la relación sujeto-verbo entre "biotadesempeña").

Además, se debe tener en cuenta el conocimiento estructurado tanto del dominio agrícola como del dominio abierto. En este caso para el primero Maraqa usa el tesauro Agrovoc (en la figura 6 aparece cada término del te- 


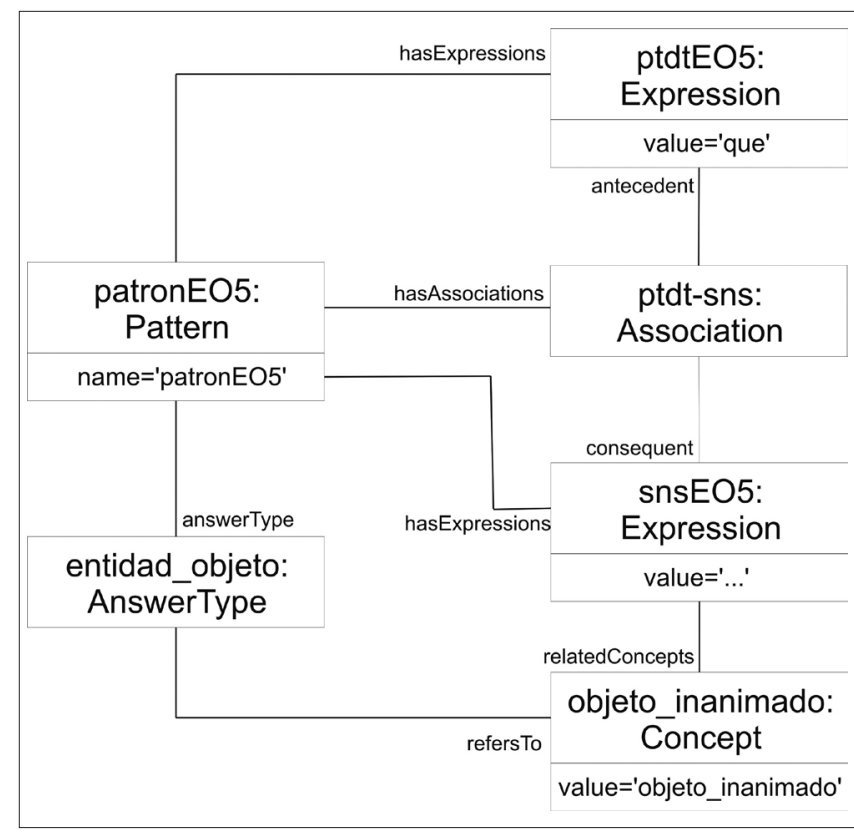

Figura 8. Ejemplo de patrones de pregunta de DA extraídos por Maraqa

restringido que estamos tratando. La figura 11 muestra la correspondiente para nuestro caso de estudio, donde se observa que el concepto "glicósidos" tiene como hiperónimo a "compuesto orgánico". Siguiendo esta taxonomía, la pregunta ejemplo tendría como TRE "glicósidos" y el espacio de búsqueda estaría sólo restringido a tipos de "glicósidos" (como "saponinas") los cuales podían ser aceptados como respuestas correctas.

Finalmente, con la taxonomía de TRE para el dominio específico, Maraqa adapta los patrones de pregunta y respuesta existentes al dominio (en este caso agrícola) y genera el código correspondiente a los mismos para ser adicionado al sistema de BR de DA. Un ejemplo de patrón de pregunta adaptado se muestra en la figura 12 y su correspondiente código en $\mathrm{C}++$ en la figura 13. Dicho patrón permite dar respuesta a la pregunta que se ha empleado de ejemplo "¿Qué glicósidos tienen un efecto defaunante en el rumen?", la cual sería "saponinas"

En la siguiente sección explicaremos cómo fueron realizados los experimentos y expondremos los resultados obtenidos.

\section{Evaluación de Maraqa en el dominio agrícola}

Para realizar la evaluación empleamos los primeros 43 volúmenes de la Revista cubana de ciencia agrícola (RCCA) como dominio restringido agrícola. Es una publicación del Instituto de Ciencia Animal, perteneciente al Ministerio de Educación Superior de la República de Cuba, editada en español e inglés desde 1967. Cada uno de los volúmenes tiene un promedio de tres o cuatro números, lo que hace un total de 140 números y 2.000 artículos $(28,65 \mathrm{MB}$ por Maraqa

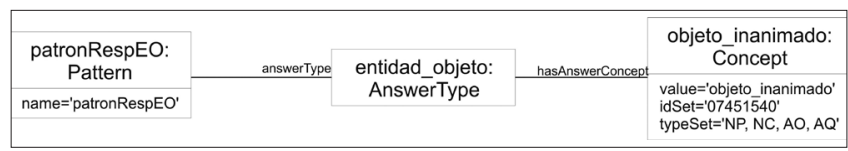

Figura 9. Ejemplo de patrones de respuesta de DA extraídos por Maraqa

como ficheros pdf). En concreto, el corpus textual RCCA está formado por un conjunto de ficheros de texto plano creados a partir de los archivos originales (con formato de texto enriquecido) de la $R C C A$, usando la utilidad de Linux pdftotext. De esta manera se evitaron los problemas con la codificación de los caracteres o con la maquetación (formato de doble columna, encabezados y pies de página, conversión de tablas y fórmulas, etc.). Para llevar a cabo los experimentos se contó con un conjunto de 330 preguntas RCCA elaboradas por expertos del dominio. La inmensa mayoría de las preguntas son de tipo factual y están basadas en hechos, preguntando por nombres de plantas, microorganismos, sustancias, fármacos, compuestos químicos, alimentos, fauna, personas, localización, día en el que sucedió algún hecho, etc. Algunos ejemplos de preguntas son: “¿Qué es la necrosis cerebrocortical?" o “¿Qué produce la cytophaga?".

Después de usar la herramienta Maraqa y los recursos de conocimiento disponibles (corpus RCCA, Agrovoc y WordNet), se obtuvo un total de 9.022 términos relevantes, 921 conceptos en la taxonomía de TRE para el dominio agrícola de la $R C C A$, y 2.600 y 325 patrones de preguntas y respuestas, respectivamente. De esta manera obtuvimos el sistema de BR para el dominio agrícola de la RCCA (Vila; Mazón; Ferrández, 2011).

Para verificar la eficiencia del sistema de BR para el dominio agrícola, obtenido a partir de AliQAn y usando Maraqa, se llevaron a cabo dos experimentos (Vila, 2010; Vila; Mazón; Ferrández, 2011). Ambos tenían como objetivo medir la precisión de los dos sistemas de BR sobre el dominio agrícola: el primer experimento usando el sistema de BR de DA AliQAn (SBR-DA AliQAn) y el segundo empleando el sistema de BR de DR agrícola (SBR-DR Agrícola). Los resultados de ambos experimentos (figura 14) demostraron que la precisión del sistema de BR de DR agrícola era mayor que la precisión

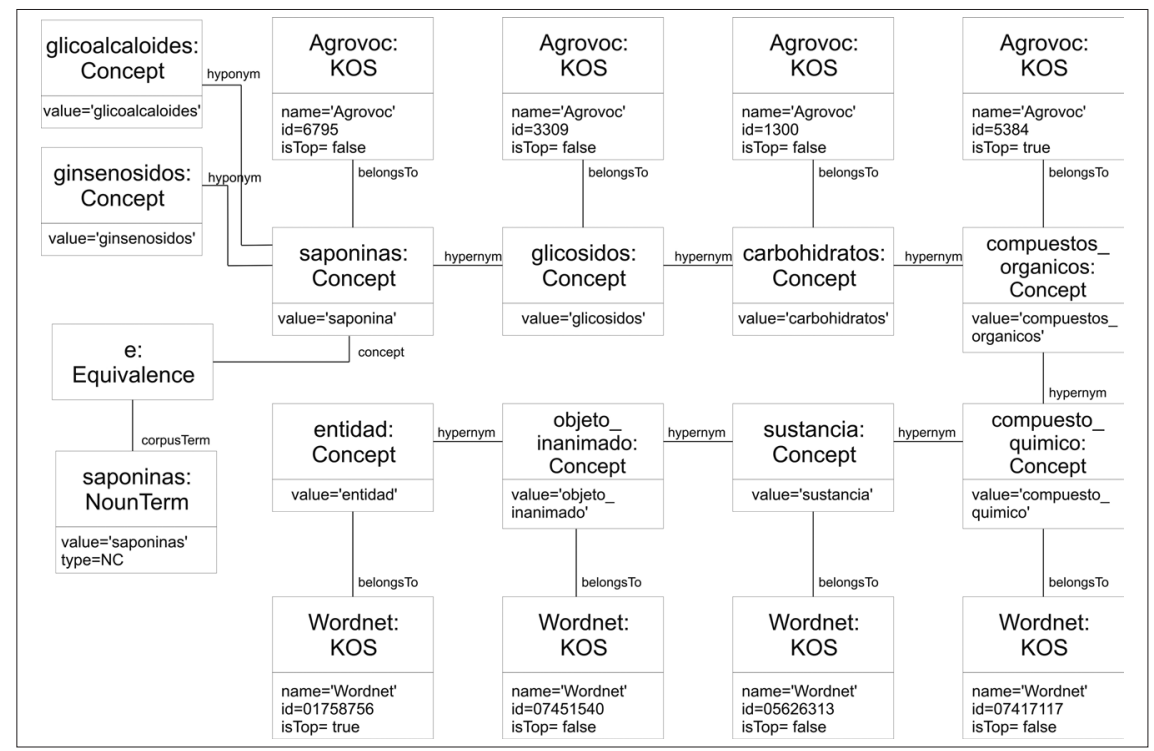

Figura 10. Ejemplo del modelo de dominio restringido y representación del concepto "saponinas" 


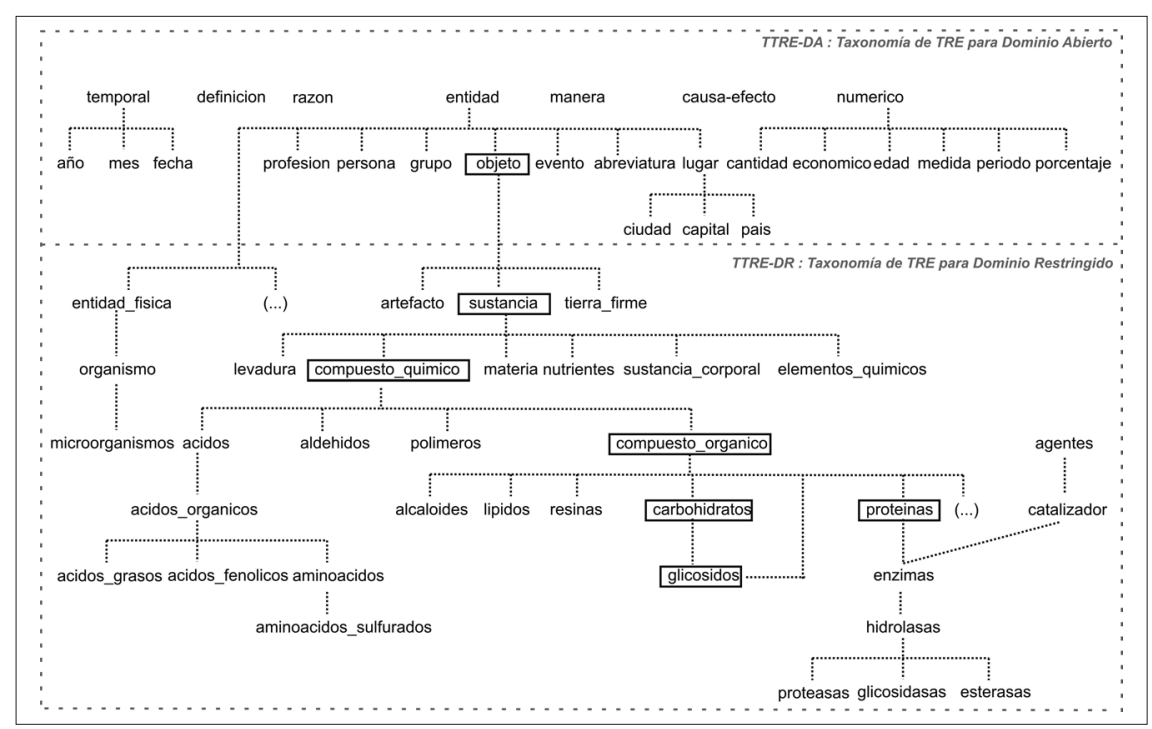

Figura 11. Ejemplo de taxonomía de TRE obtenida por Maraqa

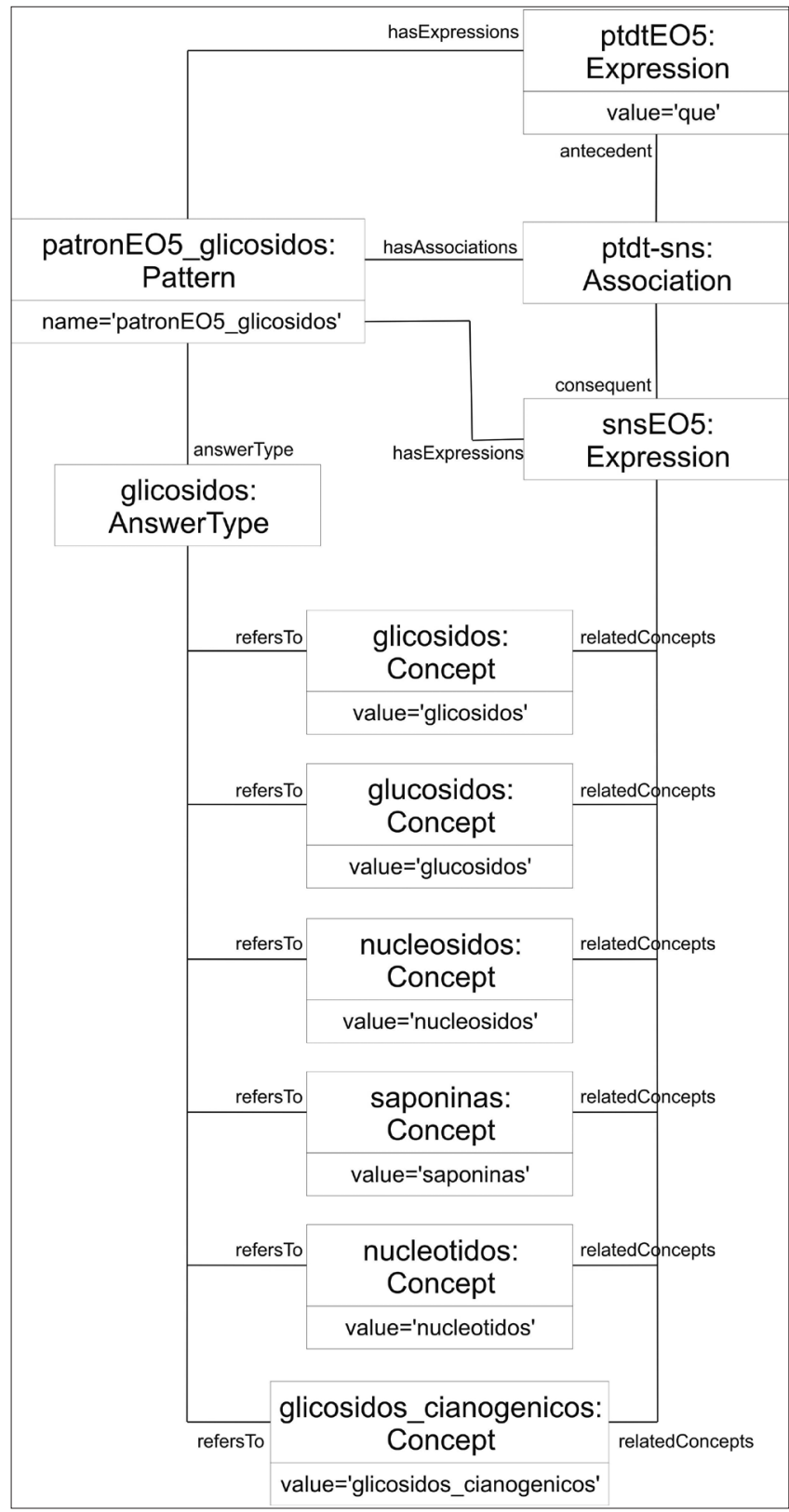

Figura 12. Ejemplo de modelo de patrón de respuesta adaptado al dominio por Maraqa del sistema de BR de DA AliQAn sobre el mismo dominio de aplicación. Por ejemplo, para las primeras respuestas del sistema baseline AliQAn sobre el dominio agrícola $R C C A$ fue de $28,8 \%$ y la del sistema de BR para el dominio agrícola fue de 58,3\%. De esta manera nuestro sistema tuvo una mejora del $29,5 \%$ de precisión en las respuestas. Estos resultados demuestran la efectividad de nuestro método que supera la media del sistema AliQAn (43\% de precisión) en todas sus actuaciones pasadas sobre dominios abiertos (Roger et al., 2008).

\section{Conclusiones}

Se ha presentado el sistema BR Mara$q a$, realizado siguiendo el paradigma del desarrollo dirigido por modelos mediante la plataforma Eclipse. Permite adaptar fácilmente sistemas de BR en DA a dominios restringidos, integrando los recursos de conocimiento anteriormente utilizados con los nuevos recursos del DR, y generando el código fuente del sistema de BR adaptado a dicho dominio.

Para ello requiere como entrada la colección de documentos sobre la que se realizará la búsqueda de información, los recursos de conocimiento del DR independientemente del formato que tengan, y el código fuente de los patrones de preguntas y respuestas del sistema de BR en DA que se desea adaptar. A partir de estos recursos, genera la taxonomía de TRE refinada para el dominio en cuestión, y el código fuente de los patrones de preguntas y respuestas adaptados al dominio. Todo el proceso de funcionamiento de la herramienta ha sido ilustrado mediante un caso de estudio en el dominio agrícola.

Con Maraqa se supera el problema de la baja precisión alcanzada cuando un sistema de BR de DA trabaja en un dominio restringido (pasando de una precisión del $28,8 \%$ al $58,3 \%)$. Además se facilita la integración de los recursos de conocimiento de dicho dominio al sistema de BR, y todo ello a partir del corpus textual como punto de partida y fuente de información principal, en detrimento del uso de corpus de preguntas de otras propuestas, lo que consideramos poco realista en DR. La principal contribución es la adaptación automática de sistemas de BR existentes (específicamente de sus patrones de pregunta-respuesta y las taxonomías de TRE) a diferentes dominios restringidos, sin requerir ningún esfuerzo manual como el resto de propuestas previas existentes (Sekine; Sudo; Nobata, 2002; Hovy; Hermjakob; Ravichandran, 2002; Metzler; Croft, 2005; Li; Roth, 2006; Ferrés; Rodríguez, 2006; Terol; Martínez-Barco; Palomar, 2006; Kosseim; Yousefi, 2008; Peñas et al., 2009).

Es posible la obtención de recursos de conocimiento (con información semántica) del dominio restringido, necesarios para aplicar Maraqa, a partir de la utilización de sistemas de alineamiento automático entre diferentes SOC's (Soergel et al., 2004; Van-Hage et al., 2010); lo que planteamos como un interesante trabajo futuro. 


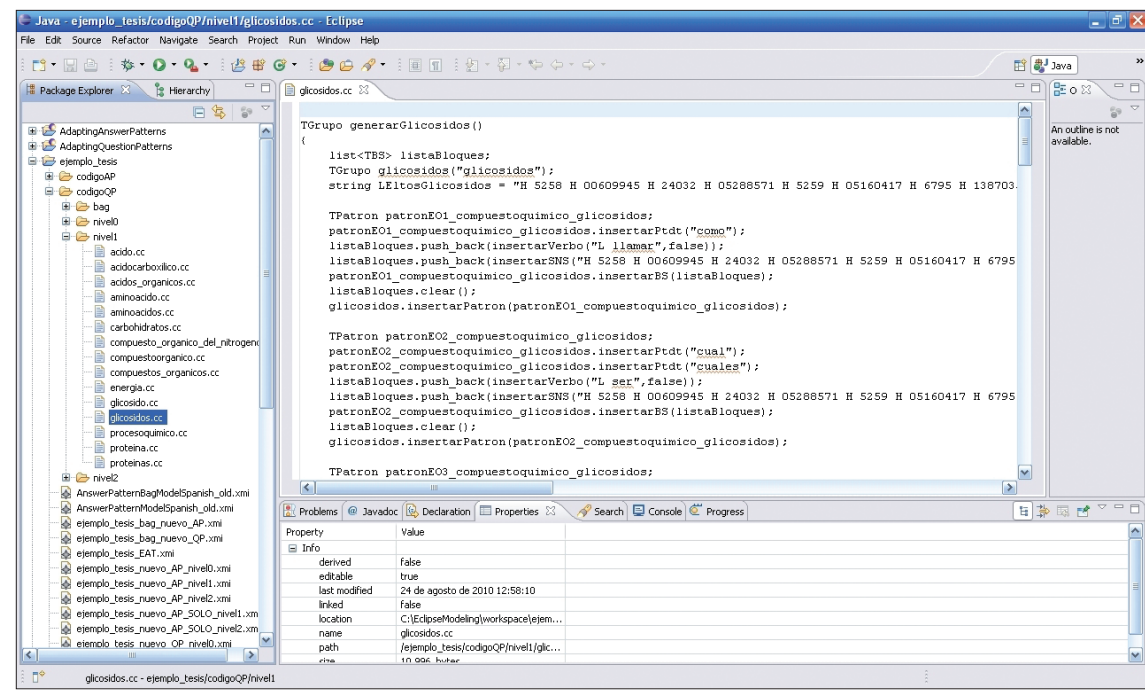

Figura 13. Ejemplo del código del patrón de pregunta adaptado al dominio por Maraqa
Ferrández, Antonio; Palomar, Manuel; Moreno, Lidia. "An empirical approach to Spanish anaphora resolution". Machine translation, 1999, v. 14, n. 3-4, pp. 191-216.

ftp://dlsi.ua.es/people/antonio/ART_ MUL5.pdf

Ferrés, Daniel; Rodríguez, Horacio. "Experiments adapting an open-domain question answering system to the geographical domain using scopebased resources". En: MLQA'06: Procs of the Workshop on multilingual question answering. Association for Computational Linguistics, 2006, pp 69-76. http://dl.acm.org/citation.cfm ?id= 1708097.1708111

Hodge, Gail. Systems of knowledge organization for digital libraries: beyond traditional authority files. The Digital Library Federation Council on Library and Information Resources, 2000. ISBN: 1887334769 http://www.clir.org/pubs/reports/pub91/pub91.pdf

Hovy, Eduard; Hermjakob, Ulf; Ravichandran, Deepak. "A question/answer typology with surface text patterns". En: Procs of the $2^{\text {nd }}$ intl conf on human language technology research, Morgan Kaufmann Publishers Inc., 2002, pp. 247-251. http://www.isi.edu/natural-language/projects/webclope dia/pubs/02hlt.pdf

Katz, Boris. "From sentence processing to information access on the World wide web". AAAl Spring symposium on natural language processing for the World wide web, 1997, pp. 77-94.

http://aaaipress.org/Papers/Symposia/Spring/1997/SS-9702/SS97-02-010.pdf

Figura 14. Precisión del sistema de BR para el dominio agrícola (desarrollado con Maraqa)

\section{Agradecimientos}

Esta investigación ha sido parcialmente financiada por el gobierno español bajo el proyecto TIN2009-13391-C04-01; y el gobierno valenciano bajo el proyecto Prometeo/2009/119. La investigación de Katia Vila ha sido financiada por una beca MAEC-Aecid del gobierno español.

\section{Bibliografía}

Acebo, S.; Ageno, Alicia; Climent, Salvador; Farreres, Javier; Padró, Lluís; Placer, Roberto; Rodríguez, Horacio; Taulé, Mariona; Turmo, Jordi. "MACO: Morphological analyzer corpus-oriented". Esprit BRA-7315 Aquilex II. Working paper 31, 1994.

Baeza-Yates, Ricardo; Ribeiro-Neto, Berthier. "Modern information retrieval". ACM Press/Addison-Wesley, 1999. ISBN: 9780321416919

Bézivin, Jean. "On the unification power of models. Software and system modeling", 2005, v. 4, n. 2, pp. 171-188. http://atlanmod.emn.fr/www/papers/OnTheUnification PowerOfModels.pdf
Katz, Boris; Borchardt, Gary; Felshin, Sue. "Natural language annotations for question answering". En: Procs of the $19^{\text {th }}$ intl Flairs conf (Flairs 2006), May 2006.

http://groups.csail.mit.edu/infolab/publications/FLAIRS 0601KatzB.pdf

Kleppe, Anneke; Warmer, Jos; Bast, Wim. MDA explained. The practice and promise of the model driven architecture. Addison Wesley, 2003. ISBN: 9780321194428

Kosseim, Leila; Yousefi, Jamileh. "Improving the performance of question answering with semantically equivalent answer patterns". Data and knowledge engineering, 2008, v. 66, n. 1, pp. 53-67.

http://dx.doi.org/10.1016/j.datak.2007.07.010

Li, Xin; Roth, Dan. "Learning question classifiers: the role of semantic information". Natural language engineering, 2006 , v. 12 , n. 3, pp. 229-249.

http://dx.doi.org/10.1017/S1351324905003955

Mellor, Stephen J.; Clark, Anthony N.; Futagami, Takao. "Model-driven development - Guest editor's introduction". IEEE software, 2003, v. 20, n. 5, pp. 14-18.

http://dx.doi.org/10.1109/MS.2003.1231145

Metzler, Donald; Croft, W. Bruce. "Analysis of statistical question classification for fact-based questions". Informa- 
tion retrieval, 2005, v. 8, n. 3, pp. 481-504.

http://ciir.cs.umass.edu/pubfiles/ir-323.pdf

http://dx.doi.org/10.1007/s10791-005-6995-3

Mollá, Diego; Vicedo, José-Luis. “Question answering in restricted domains: an overview". Computational linguistics, 2007, v. 33, n. 1, pp. 41-61.

http://www.mitpressjournals.org/doi/pdf/10.1162/coli. 2007.33.1.41

http://dx.doi.org/10.1162/coli.2007.33.1.41

Peñas, Anselmo; Forner, Pamela; Sutcliffe, Richard; Rodrigo, Álvaro; Forascu, Corina; Alegria, Iñaki; Giampiccolo, Danilo; Moreau, Nicolas; Osenova, Petya. "Overview of ResPubliQA 2009: question answering evaluation over European legislation". En: Working notes of Cross language evaluation forum (CLEF), 2009.

http://clef.isti.cnr.it/2009/working_notes/ResPubliQAoverview.pdf

Roger, Sandra; Vila, Katia; Ferrández, Antonio; Pardiño, María; Gómez, José-Manuel; Puchol-Blasco, Marcel; Peral, Jesús. "Using AliQAn in monolingual QA@Clef 2008". 9 $9^{\text {th }}$ Workshop of the Cross-language evaluation forum. Lecture notes in computer science, 2008, v. 5706, pp. 333-336. http://dx.doi.org/10.1007/978-3-642-04447-2_38

Russell, Stuart; Norvig, Peter. "Artificial intelligence: a modern approach". Pearson education, $2^{\text {nd }}$ ed., 2003. ISBN: 978 0137903955

Sekine, Sstoshi; Sudo, Kiyoshi; Nobata, Chikashi. "Extended named entity hierarchy". En: Procs of $3^{\text {rd }}$ Intl conf on lan- guage resources and evaluation (LREC'02), 2002, pp. 18181824.

http://nlp.cs.nyu.edu/pubs/papers/sekine-Irec02.pdf

Soergel, Dagobert; Lauser, Boris; Liang, Anita; Fisseha, Frehiwot; Keizer, Johannes; Katz, Stephen. "Reengineering thesauri for new applications: the Agrovoc example". Journal of digital information, 2004, v. 4, n. 4.

http://journals.tdl.org/jodi/article/viewArticle/112/111

Terol, Rafael M.; Martínez-Barco, Patricio; Palomar, Manuel. "Aplicación de técnicas basadas en PLN al tratamiento de preguntas médicas en búsqueda de respuestas". Procesamiento del lenguaje natural, 2006, n. 36, pp. 17-24. http://www.sep/n.org/revistaSEPLN/revista/36/02.pdf

Van-Hage, Willem-Robert; Sini, Margherita; Finch, Lori; Kolb, Hap; Schreiber, Guus. "The OAEI food task: an analysis of a thesaurus alignment task". Applied ontology, 2010, v. 5, n. 1 , pp. 1-28.

http://dx.doi.org/10.3233/AO-2010-0072

Vila, Katia. Búsqueda de respuestas en dominios restringidos: aplicación sobre el dominio agrícola. Tesis doctoral, Universidad de Alicante, 2010.

http://rua.ua.es/dspace/bitstream/10045/18329/1/tesis_ vila.pdf

Vila, Katia; Mazón, José-Norberto; Ferrández, Antonio. "Model-driven development for adapting question answering systems to restricted domains". Journal of research and practice in information technology, May 2011, v. 43, n. 2, pp. 23-40.

http://dx.doi.org/10.1145/1966883.1966893

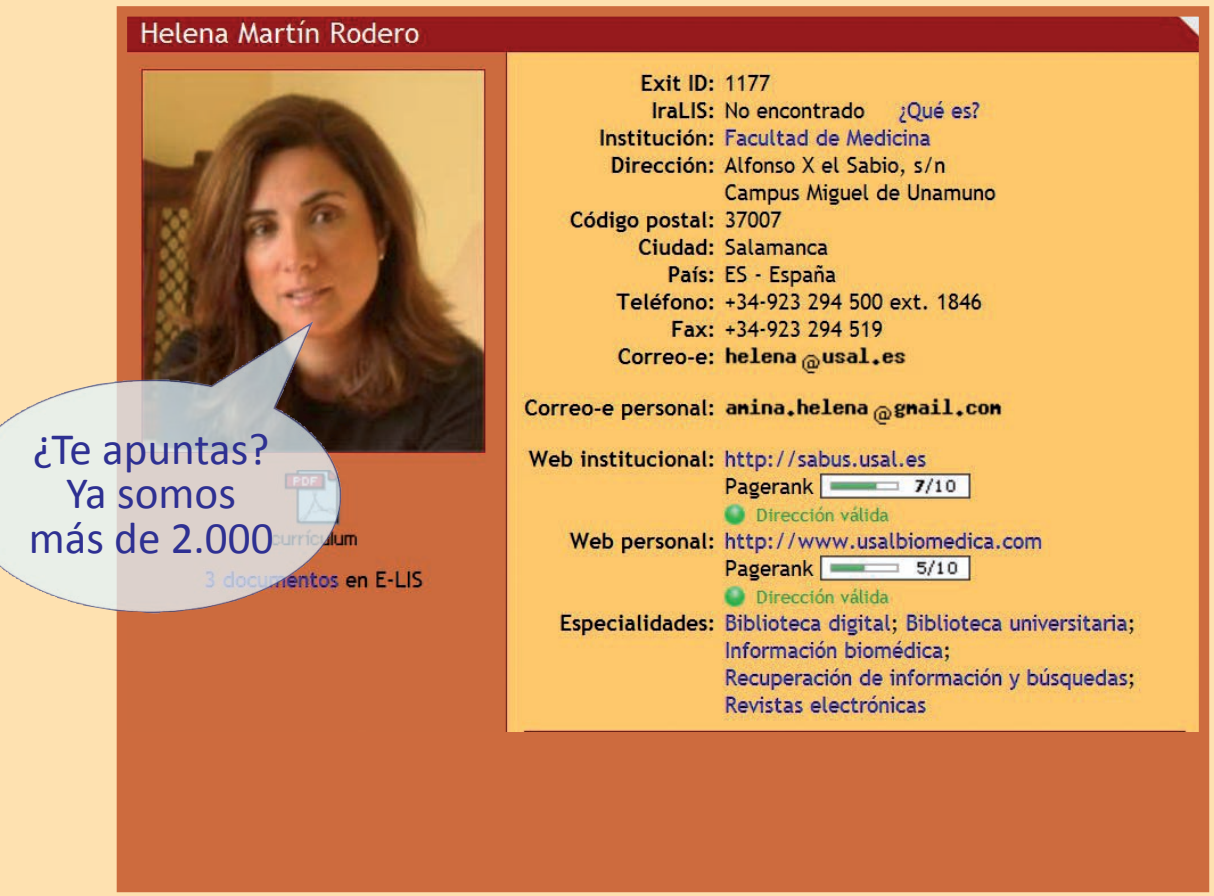

http://directorioexit.info
Para titulados con más de 1 año de experiencia, que hayan publicado algún artículo o ponencia o puedan dar clase más de 1 hora. 